\title{
Relationship Between Arm Span Measurements and Body Height in Plav
}

\author{
Milos Kovacevic ${ }^{1}$, Jovan Radulovic ${ }^{1}$ \\ 'University of Montenegro, Faculty for Sport and Physical Education, Niksic, Montenegro
}

\begin{abstract}
The unusual height of Montenegrin inhabitants of the highland region has been recognised by European anthropologists more than 100 years ago. In light of rather sparse recent scientific literature, the purpose of this research study was to examine the body height in both sexes of Montenegrin adults nowadays. Furthermore, the relationship between arm span and body height, which varies in different ethnic and racial groups, was used as an alternative to estimating the body height for some groups of the population. The nature and scope of this study analyses 29 adolescents ( 14 men and 15 women) from Plav. The anthropometric measurements were taken according to the protocol of the International Society for the Advancement of Kinanthropometry (ISAK). Means and standard deviations were obtained. A comparison of means of body heights and arm spans within and between the sexes were carried out using a t-test. The relationships between body height and arm span were determined using simple correlation coefficients and their $95 \%$ confidence interval. A linear regression analysis was then performed to examine the extent to which arm span can reliably predict body height. The results have shown that male from Plav are $180.80 \pm 8.23$ centimetres tall and have an arm span of $180.51 \pm 8.26$ centimetres, while female from Plav are $163.00 \pm 6.33$ centimetres tall and have an arm span of $164.12 \pm 5.94$ centimetres. Comparing the results with other studies has shown that both sexes of Plav are very tall.
\end{abstract}

Key words: Prediction, Standing Height, Arm Span, Kolasin

\section{Uvod}

Crna Gora se nalazi u jugoistočnoj Evropi, na Balkanskom poluostrvu. Teritorija Crne Gore zauzima približno 13.812 $\mathrm{km}^{2}$. Graniči se sa Hrvatskom (14 km duga granica) na zapa$\mathrm{du}$, Bosnom i Hercegovinom na sjeveru (225 km), Srbijom na istoku $(203 \mathrm{~km})$, Albanijom na jugu $(172 \mathrm{~km})$, a od Italije je razdvojena Jadranskim morem. Dužina obale je $293.5 \mathrm{~km}$. Crna Gora je demokratska i multietnička republika. Osnovna etnička struktura Crne Gore je: 44,98\% čine Crnogorci, 28,73\% Srbi, 8,65\% su Bošnjaci i 4,91\% Albanci (Bjelica, Popović, Kezunović, Jurak, i Grasgruber, 2012). Geografski gledano, dijeli se na tri regije: planinski dio, centralni dio i primorje. Ovo istraživanje analizira odnos tjelesne visine i raspona ruku kod građana Plava, koji se teritorijalno nalaze na istočnom dijelu države i pripadaju sjevernoj regiji, što je i prikazano na slici 1. Plav je gradsko naselje i sjedište istoimene opštine. Prema popisu iz 2011. godine imao je 3.717 stanovnika. Nalazi se u podnožju planinskog masiva Prokletije, na rijeci Lim. Površina opštine Plav iznosi oko $486 \mathrm{~km}^{2}$. Istraživanja, koja su sproveli evropski antropolozi prije jednog vijeka, dokazala su pretpostavku da najvisočiji ljudi žive na prostoru Dinaridia (Pineau et al., 2005).

Dinarski Alpi prepoznati su kao područje sa visokom populacijom od evropskih antropologa prije više od 100 godina (Bjelica et al., 2012; Masanovic, 2018a; Gardašević, Rašidagić, Krivokapić, Ćorluka, i Bjelica, 2017; Pineau, Delamarche, \& Božinović, 2005). Imajući u vidu da savremeni Crnogorci, kao i drugi narodi sa prostora bivše Jugoslavije, spadaju više u dinarsku rasnu klasifikaciju nego u bilo koju drugu, istraživači ove studije pretpostavljali su da adolescenti sa prostora opštine Plav mogu biti malo viši ili jednako visoki kao i najviši stanovnici Crne Gore, samim tim bi se našli u društvu najviših nacija u Evropi.

\section{Correspondence:}

\section{Montenegro J.Radulovic}

Sport University of Montenegro, Faculty for Sport and Physical Education, Narodne omladine bb, 81400, Niksic, Montenegro E-Mail: Jovanradulovicradule@gmail.com 


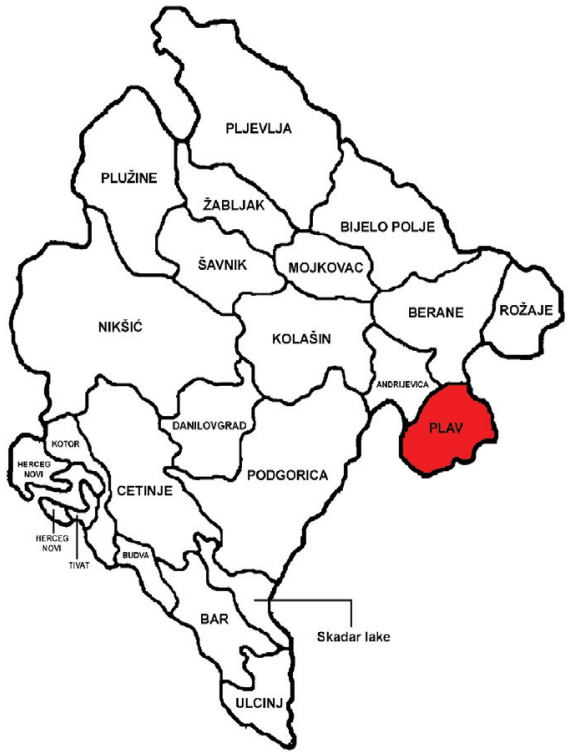

Slika. 1. Teritorija Plava

Neuobičajna visina Crnogoraca je činjenica koja je primjećena od evropskih antropologa prije više od 100 godina. Uzorak od 800 crnogorskih muškaraca koji je mjerio Robert V. Ehrich početkom 20. vijeka davao je najveći prosijek u čitavoj Evropi $177 \mathrm{~cm}$, dok su neke oblasti imale tendenciju ka178 centimetara (Bjelica et al., 2012; Popović, 2016). U međuvremenu je sproveden veliki broj istraživanja te Crnogorci sada nisu najviši, ali su i dalje u samom vrhu.

Naučna literatura nam je pokazala da je mjerenje tjelesne visine izuzetno važna varijabla kada se procjenjuje nutritivni status (Arifi et al., 2017; Datta Banik, 2011), kada se procjenjuje rast djece, kada je potrebno ocjenjivanje osnovnih energetskih zahtjeva, prilagođavanje mjera fizičkog kapaciteta i predviđanje doziranja ljekova i postavljanje standarda fizioloških varijabli (npr. snaga mišića, brzina metabolizma, zapremine pluća i glomerularna filtracija). Međutim, tačan stepen ne može uvjek biti identifikovan i riješen na uobičajan način zbog raznih remetećih faktora kao što su na primer, paraliza, frakture, amputacija, skolioza i bol (Quanjer et al., 2014). Zbog ovog faktora, procjena tjelesne visine mora se dobiti na osnovu drugih pouzdanih antropometrijskih indikatoria kao što su dužina ruku i stopala (Agnihotri, Purwar, Googoolybe, Agnihotri, \& Jeebun, 2007; Arifi et al., 2017; Masanovic, Gardasevic, i Arifi, 2018a; Masanovic, Gardasevic, i Arifi, 2018b; Masanovic, Gardasevic, i Arifi, 2018c), visina koljena (Arifi et al., 2017; Karadag, Ozturk, Sener, i Altuntas, 2012), dužina podlaktice (Arifi et al., 2017), dužina grudnog koša (Arifi et al., 2017; Menezes et al., 2009), dužine kičmenog stuba (Arifi et al., 2017; Nagesh \& Pradeep Kumar, 2006), sjedeća visina (Arifi et al., 2017; Gardasevic, 2018a; Masanovic, 2018b; Masanovic, Gardasevic, i Arifi, 2018d: Masanovic, Gardasevic, i Arifi, 2018e; Masanovic, Gardasevic, i Arifi, 2018f; Masanovic, Gardasevic, i Arifi, 2018g), dužina lopatice (Arifi et al., 2017; Campobasso et al., 1998), dužine tibije (Masanovic, 2018c; Gardasevic, 2018b; Gardasevic, Masanovic, i Arifi, 2018a; Gardasevic, Masanovic, i Arifi, 2018b; Gardasevic, Masanovic, i Arifi, 2018c), raspon ruku (Arifi et al., 2017; Bjelica i sar., 2012; Popović i Bjelica, 2016; Popović, Bjelica, Milašinović, Gardašević i Rašidagić, 2016; Dragutinovic, 2018; Dragutinovic, 2018a; Knezevic, 2018; Vukotic, 2018). Prema tome, svi navedeni antropometrijski indikatori, koji se koriste kao alternativa za procjenu relativne tjelesne visine, veoma su važni u svim slučajevima gore pobrojanim, a u kojima je tjelesna visina značajna te se ne može izmjeriti standardnom metodom. Takođe, važno je istaći da sve navedeno treba i mora primijeniti u sportskim naukama, budući da važnost tjelesne visine koja utiče na uspjeh u različitim sportskim disciplinama (Popović, 2017). Značajan je broj istraživanja koja su upućivala na korisnost primjenjivanja različitih tjelesnih parametara u procjenjivanju relativne tjelesne visine (Mašanović, 2017; Popovic, Arifi, \& Bjelica, 2017; Popović i Bjelica, 2017; Popovic, Gardasevic, Masanovic, Arifi, \& Bjelica, 2017, Kovacevic, 2018; Kovacevic, 208a; Knezevic, 2018a; Gardasevic, Masanovic, \& Arifi, 2018; Arifi, Gardasevic, \& Masanovic, 2018), a ispostavilo se da je raspon ruku, upravo najpouzdaniji od svih ostalih (Masanovic, 2017).

$\mathrm{Na}$ osnovu dosadašnjih istraživanja, autori ovog rada su se bazirali na crnogorsku teritoriju, te s toga, cilj ovog istraživanja bio je da se ispita tjelesna visina kod oba pola adolescenata opštine Plav, kao i da se utvrdi da li raspon ruku može biti adekvatna alternativna mjera za utvrđivanje relativne tjelesne visine.

\section{Metod rada}

U ovom istraživanju učestvovalo je ukupno 29 ispitanika (14 muškog i 15 ženskog pola) iz opštine Plav. Starost ispitanika kompletnog uzorka iznosi 17.80 \pm 0.41 . Raspon u godinama ispitanika bio je od 17 do 20 godina. Ključni kriterijumi za prihvatanje uzorka ispitanika muškog i ženskog pola bili su da: ispitanik dobrovoljno učestvuje $u$ radu i da živi na prostoru opštine Plav.

Analiza je izvršena korišćenjem statističkog paketaza društvene nauke (SPSS) verzija 20.0. Za obije antropometrijske varijable obrađeni u centralni i disperzivni parametri u okviru osnovne statistike i to: raspon (minimalna i maksimalna vrijednost), aritmetička sredina i standardna devijacija. T-testom su provjerene razlike aritmetičkih sredina antropometrijskih varijabli u odnosu na pol ispitanika. Veza između tjelesne visine i raspona ruku je obrađena korelacionom analizom s pouzdanošću od 95\%. Linearnom regresionom analizom je utvrđena predikcija raspona ruku na kriterijsku varijablu tjelesna visina.

\section{Rezultati}

Rezultati centralnih i disperzivnih parametara za ispitanike oba pola nalaze se u tabeli 1 . Aritmetička sredina varijable

Tabela 1. Centralni i Disperzivni parametri antropometrijskih karakteristika

\begin{tabular}{ccc}
\hline Ispitanici & $\begin{array}{c}\text { tjelesna visina } \\
(\mathbf{A S} \pm \text { SD) }\end{array}$ & $\begin{array}{c}\text { raspon ruku } \\
(\mathbf{A S} \pm \text { SD) }\end{array}$ \\
\hline \multirow{2}{*}{ Muški } & $170.2-195.2$ & $170.1-195.5$ \\
& $(180.80 \pm 8.23)$ & $(180.51 \pm 8.26)$ \\
\multirow{2}{*}{ Ženski } & $152.8-173.0$ & $152.5-173.1$ \\
& $(163.00 \pm 6.33)$ & $(164.12 \pm 5.93)$ \\
\hline
\end{tabular}


tjelesna visina, kod ispitanika muškog pola iznosi $180.80 \pm 8.23$ $\mathrm{cm}$, a varijable raspon ruku $180.51 \pm 8.26 \mathrm{~cm}$, što znači da je aritmetička sredina raspona ruku za $0.29 \pm 0.03 \mathrm{~cm}$ manja. Kod ispitanika ženskog pola aritmetička sredina varijable tjelesna visina iznosi $163.00 \pm 6.33 \mathrm{~cm}$, a varijable raspon ruku $164.12 \pm 5.93 \mathrm{~cm}$, što znači da je aritmetička sredina raspona ruku za $1.12 \pm 0.40 \mathrm{~cm}$ viša.

$\mathrm{U}$ tabeli 2 prikazani su rezultati korelacione analize izme- đu tjelesne visine i raspona ruku s pouzdanošću od 95\% za ispitanike oba pola. Povezanost, odnosno korelacija između tjelesne visine i raspona ruku je statistički značajna na nivou 0.000 i kod ispitanika muškog i kod ispitanika ženskog pola. Koeficijenti korelacije su veoma visoki i kod ispitanika muškog pola taj koeficijent iznosi 0.989, a kod ispitanika ženskog pola 0.953 .

Tabela 2. Korelaciona analiza između tjelesne visine i raspona ruku

\begin{tabular}{cccc}
\hline Ispitanici & Koeficijent korelacije & $\begin{array}{c}\text { Interval pouzdanosti } \\
\mathbf{9 5 \%}\end{array}$ & Nivo značajnosti \\
\hline Muški & 0.989 & $0.968-1.000$ & 0.000 \\
Ženski & 0.953 & $0.893-0.992$ & 0.000 \\
\hline
\end{tabular}

Rezultati linearne regresione analize su prikazani u tabeli 3. Koeficijent regresije kod ispitanika oba pola je istovjetan $s$ koeficijentom regresije iz prethodnih analiza, i veoma visok. Nivo značajnosti kod ispitanika oba pola iznosi 0.000 , što zna- či da je raspon ruku adekvatna alternativna mjera tjelesnoj visini, tj. u odnosu na raspon ruku može se predvidi tjelesna visina kod ispitanika oba pola opštine Plav. To potvrđuju i koeficijenti determinacije, gdje za muškarce taj koeficijent

Tabela 3. Rezultati linearne regresione analize (predikcija raspona ruku na tjelesnu visinu)

\begin{tabular}{cccccc}
\hline Ispitanici & $\begin{array}{c}\text { Koeficijent } \\
\text { regresije }\end{array}$ & $\begin{array}{c}\text { Standardna } \\
\text { greška }\end{array}$ & $\begin{array}{c}\text { Koeficijent } \\
\text { determinacije \% }\end{array}$ & t vrijednost & $\begin{array}{c}\text { Nivo } \\
\text { značajnosti }\end{array}$ \\
\hline Muški & 0.989 & 1.295 & 97.7 & 22.656 & 0.000 \\
Ženski & 0.953 & 1.984 & 90.9 & 11.392 & 0.000 \\
\hline
\end{tabular}

objašnjava $97.70 \%$ varijabiliteta, a kod djevojaka $90.90 \%$ varijabiliteta, pa samo mali procenat ostaje neobjašnjen, odnosno pod uticajem drugih faktora koji nijesu bili obuhvaćeni ovim istraživanjem.
Parametri tjelesne visine i raspona ruku, kao i njihova veza prikazani su udruženo za ispitanike oba pola pomoću skater dijagrama (dijagram 1).

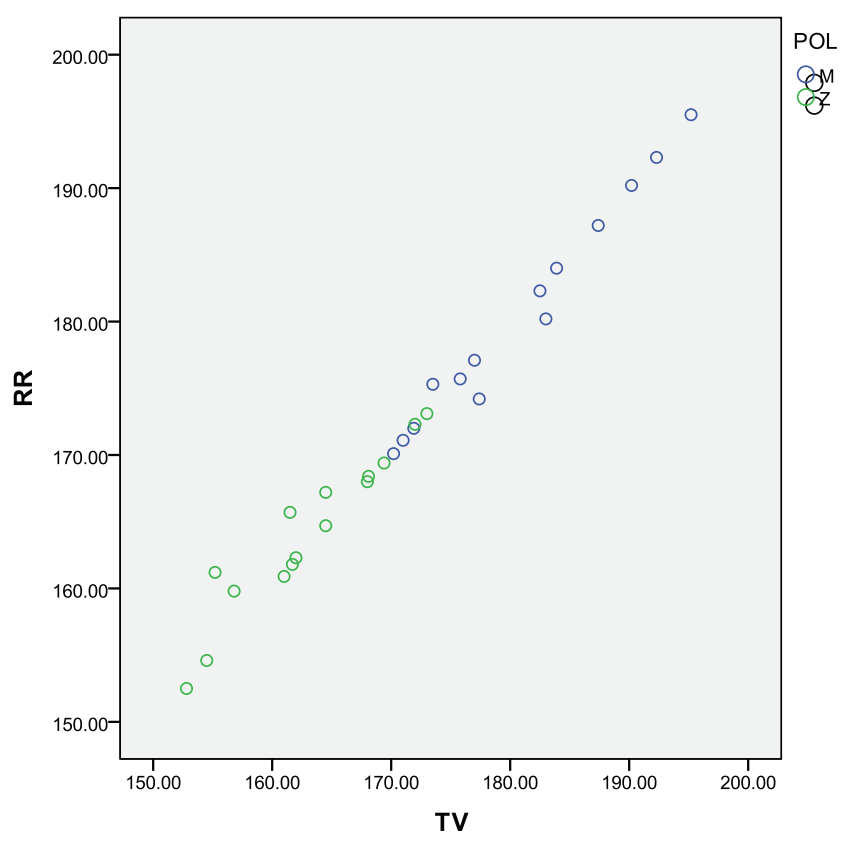

Slika 2. Skater dijagram i veza između raspona ruku i tjelesne visine među oba pola

\section{Diskusija}

Ovim istraživanjem dobijeni su podaci koji pokazuju prosječnu visinu kod adolescenata oba pola građana opštine Plav i mogu biti korisni za upoređivanje sa drugim gradovima Crne Gore, izračunavanje prosjeka na nivou sjeverne regije kao i kompletne države. Rezultati su pokazali da su muškarci sa prostora opštine Plav, prosječno visoki $180.80 \mathrm{~cm}$, dok im je prosječan raspon ruku $180.51 \mathrm{~cm}$. Dakle, uzimajući u obzir ovaj pokazatelj možemo zaključiti da su muškarci sa teritorije opštine Plav niži od prosjeka koji važi za kompletnu mušku populaciju u Crnu Goru i iznosi $183.21 \mathrm{~cm}$, dok je respon ruku takođe niži od prosjeka koji je utvrđen na nivou Crne Gore, a koji iznosi $185.71 \mathrm{~cm}$ (Bjelica et al., 2012). Takođe, niži su od prosjeka koji važi za sjevernu regiju Crne Gore, koji iznosi 
$183.29 \mathrm{~cm}$, a raspon ruku je takođe niži od prosječnog sjeverne regije koji iznosi $184.29 \mathrm{~cm}$ (Popović, Bjelica, Milašinović i Gardašević, 2016; Milašinović, Gardašević, i Bjelica 2017). Niži su od Bosanaca $183.9 \mathrm{~cm}$ (Popović i sar., 2015), Srba 182.0 cm (Popović i sar., 2013), Litvanca $180.6 \mathrm{~cm}$ (Tutkuviene, 2005), Islanđana $180.6 \mathrm{~cm}$ (Dagbjartsson i sar., 2000), Hrvata $180.5 \mathrm{~cm}$ (Juresa i sar., 2012), Slovenaca $180.3 \mathrm{~cm}$ (Starc, \& Strel, 2011), Čeha (Vignerova i sar., 2006). Popović (2017) sproveo je istraživanje, kako bi ispitao prosječnu tjelesnu visinu u opštinama Crne Gore i došao je do zanimljivih podataka. Prosječna visina Kolašinaca i Šavničana iznosi preko 185 cm, dok su u Danilovgradu, Nikšiću, Plužinama i Žabljaku, muškarci u prosjeku visoki preko $184 \mathrm{~cm}$. Nasuprot tome, najniži Crnogorci koji u većoj mjeri umanjuju prosjek na nivou kompletne države, žive u Plavu, Andrijevici i na Cetinju, sa prosječnom visinom koja iznosi $181 \mathrm{~cm}$.

Kada su u pitanju adolescentkinje nastanjene na teritoriji opštine Plav, njihova prosječna tjelesna visina iznosi 163.00 $\mathrm{cm}$, pa se može konstatovati da kao i njihovi sugrađani, spadaju među najniže u Crnoj Gori, a samim tim i u Evropi. Ako se uporedi prosječna visina adolescentkinja opštine Plav sa prosjekom koji važi za sjevernu regiju Crne Gore dolazi se do saznanja da su Plavljanke niže od prosjeka koji je dobijen za sjevernu regiju i iznosi $168.96 \mathrm{~cm}$. Isti je slučaj i sa prosječnim rasponom ruku adolescentkinja sjeverne regije koji iznosi $167.71 \mathrm{~cm}$ (Milašinović, Popović, Bjelica, i Vasiljević, 2016). U poređenju sa relevantnim istraživanjima, ispred njih su se našle djevojke iz Bosne $171.8 \mathrm{~cm}$ (Popović i sar., 2015) i Holandije $168.8 \mathrm{~cm}$ (Statistics Netherlands, 2016).

Imajući u vidu istraživanje koje je sprovedeno na teritoriji opštine Tivt, na osnovu koga je utvrđeno da prosječna tjelesna visina adolescenata te poštine iznosili $182.36 \mathrm{~cm}$, može se konstatovati da je prosječna tjelesna visina adolescenata opštine Plava od $180.80 \mathrm{~cm}$ značajno niža. Kada su u pitanju adolescentkinje opštine Plav, njihova prosječna tjelesna visina iznosi $163.00 \mathrm{~cm}$, za razliku od adolescentkinja opštine Tivat, čija prosječna tjelesna visina iznosi $168.96 \mathrm{~cm}$, što govori da je i kod ženske populacije poredivši ova dva grada, značajna razlika. Kada je u pitanju raspon ruku, kod adolescenata opštine Plav utvrđeno je da iznosi prosječno $180.51 \mathrm{~cm}$, dok kod adolescenata opštine Tivt raspon ruku prosječno iznosi $180.79 \mathrm{~cm}$, pa se može konstatovati da raspon ne pokazuje tolike razlike kao tjelesna visina. Kada je u pitanju raspon ruku kod djevojka u obije opštine, djevojkama iz Plava izračunata je vrijednost od $164.12 \mathrm{~cm}$, dok je kod djevojaka iz Tivta raspon ruku iznosio $166.15 \mathrm{~cm}$, pa se može uočiti da postoji značajna razlika između ovih pokazatelja. Postojanje ovakvih varijacija nam potvrđuje potrebu za izradom posebnih modela visine za svaki dio u Crnoj Gori (Milašinović i sar., 2016).

Takođe u obzir se mora uzeti i činjenica da su u ovom istraživanju učestvovali adolescenti, što odmah navodi na činjenicu da njihov rast nije u potpunosti završen. Rezultati su nam pokazali da raspon ruku kod adolescenata opštine Plav, jeste adekvatna alternativna mjera tjelesnoj visini. Naredna istraživanja bi trebalo sprovoditi po principu regionalne podjele države, odnosno da se na taj način utvrdi da li postoje razlike u odnosu na geografko područje, koje značajno utiču na prosječnu tjelesnu visinu kompletne populacije, kod oba pola, ali i njenu povezanost sa rasponom ruku. Ovo istzraživanje može poslužiti kao još jedna baza, za dalje naučne radove koji će se baviti ovom problematikom u cilju dolaska do relevantnih informacija, te samim tim unaprijeđenja nauke.

\section{Acknowledgements}

There are no acknowledgements.

\section{Conflict of Interest}

The authors declare that there are no conflicts of interest.

Received: 13 September 2018 | Accepted: 11 October 2018 | Published: 29 October 2018

\section{References}

Agnihotri, A. K., Purwar, B., Googoolybe, K., Agnihotri, S., \& Jeebun, N. (2007). Estimation of stature by foot length. J. Forensic Leg. Med. 14(5), 279-83.

Arifi, F., Gardasevic, J., \& Masanovic, B. (2018). Relationship between foot length measurements and body height: $A$ prospective regional study among adolescents in central region of Kosovo. Sport Mont, 16(3), 7579. doi: $10.26773 / \mathrm{smj} .181013$

Arifi, F., Bjelica, D., Sermaxhaj, S., Gardasevic, J., Kezunovic, M., \& Popovic, S. (2017). Stature and its Estimation Utilizing Arm Span Measurements in Kosovan Adults: National Survey. International Journal of Morphology, 35(3), 1161-7.

Bjelica, D., Popovic, S., Kezunovic, M., Petkovic, J., Jurak, G. \& Grasgruber, P. (2012). Body Height and Its Estimation Utilizing Arm Span Measurements in Montenegrin Adults. Anthropological Noteboos, 18(2), 69-83.

Campobasso, C. P., Di Vella, G., \& Introna, F. Jr. (1998). Using scapular measurementsinregressionformulaefortheestimation of stature.Boll.Soc.Ital.Biol. Sper. 74(7-8)75-82.

Datta Banik, S. (2011). Arm span as a proxy measure for height and estimation of nutritional status: a study among Dhimals of Darjeeling in West Bengal India. Ann. Hum. Biol. 38(6), 728-35.

Dragutinovic, K. (2018). Relationship between arm span measurements and body height in Herceg Novi. Journal of Anthropology of Sport and Physical Education, 2(3), 27-32. doi: 10.26773/jaspe.180705

Dragutinovic, K. (2018a). Relationship between arm span measurements and body height in Bar. Journal of Anthropology of Sport and Physical Education, 2(3), 111-115. doi: 10.26773/jaspe.180719

Gardasevic, J. (2018a). Standing Height/Sitting Height Ration in Eastern-Kosovan Adolescents. Retrieved from SSRN's eLibrary: https://ssrn. com/abstract $=3141566$

Gardasevic, J. (2018b). Standing Height/Tibia Length Ration in Western-Kosovan Adolescents. Retrieved from SSRN's eLibrary: https://ssrn. com/abstract $=3138101$

Gardasevic, J., Masanovic, B., \& Arifi, F. (2018). Relationship between tibia length measurements and standing height: A prospective regional study among adolescents in southern region of Kosovo. Sport Mont, 16(3), 51-55. doi: 10.26773/smj.181009

Gardasevic, J., Masanovic, B., Arifi, F. (2018a). Relationship Tibia Length/ Standing Height in Central-Kosovan Adolescents. Retrieved from SSRN's eLibrary: https://ssrn.com/abstract $=3138122$

Gardasevic, J., Masanovic, B., Arifi, F. (2018b). Relationship Tibia Length/ Standing Height in Northern-Kosovan Adolescents. Retrieved from SSRN's eLibrary: https://ssrn.com/abstract=3138112

Gardasevic, J., Masanovic, B., Arifi, F. (2018c). Relationship Tibia Length/ Standing Height in Southern-Kosovan Adolescents. Retrieved from SSRN's eLibrary: https://ssrn.com/abstract=3138105

Gardasevic, J., Rasidagic, F., Krivokapic, D., Corluka, M., \& Bjelica, D. (2017). Stature and Its Estimation Utilizing Arm Span Measurements in Male Adolescents from Herzeg-Bosnia Entity in Bosnia and Herzegovina. Montenegrin Journal of Sports Science and Medicine, 6(1), 37-44.

Karadag, B., Ozturk, A. O., Sener, N., \& Altuntas, Y. (2012) Use of knee height for the estimation of stature in elderly Turkish people and their relationship with cardiometabolic risk factors. Arch. Gerontiol. Germatr. 54(1), 82-9.

Knezevic, M. (2018). Relationship between arm span measurements and body height in Bijelo Polje. Journal of Anthropology of Sport and Physical Education, 2(3), 143-147. doi: 10.26773/jaspe.180725

Knezevic, M. (2018a). Relationship between arm span measurements and body height in Mojkovac. Journal of Anthropology of Sport and Physical Education, 2(3), 165-169. doi: 10.26773/jaspe.180729

Kovacevic, M. (2018). Relationship between arm span measurements and body height in Danilovgrad. Journal of Anthropology of Sport and Physical Education, 2(3), 33-37. doi: 10.26773/jaspe.180706

Kovacevic, M. (2018a). Relationship between arm span measurements and body height in Kolasin. Journal of Anthropology of Sport and Physical Education, 2(3), 149-153. doi: 10.26773/jaspe.180726

Masanovic, B. (2017). Relationship between arm span measurements and body height in Dinaric Alpes population: A systematic review. Journal of Anthropology of Sport and Physical Education, 1(1), 33-7.doi: 10.26773/ jaspe.171006 
Masanovic, B. (2018a). Standing Height and its Estimation Utilizing Arm Spam and Foot Length Measurements in Dinaric Alps Population: A Systematic Review. Sport Mont 16(2), 1-6.

Masanovic, B. (2018b). Standing Height/Sitting Height Relationhip in Western Region in Kosovo. Retrieved from SSRN's eLibrary: https://ssrn com/abstract=3138518

Masanovic, B. (2018c). Tibia Length and Standing Heigh Relationshipt in Eastern Region of Kosovo. Retrieved from SSRN's eLibrary: https://ssrn com/abstract $=3143118$

Masanovic, B., Gardasevic, J., \& Arifi, F. (2018a). Relationship between foot length measurements and body height: A prospective regional study among adolescents in eastern region of Kosovo. Sport Mont, 16(1), 9-13. doi: $10.26773 /$ smj.180202

Masanovic, B., Gardasevic, J., \& Arifi, F. (2018b). Relationship between Foot Length Measurements and Body Height: A Prospective Regional Study among Adolescents in Northern Region of Kosovo. Anthropologie-International Journal of Human Diversity and Evolution, in pres, https:// doi.org/10.26720/anthro.18.01.23.1

Masanovic, B., Gardasevic, J., \& Arifi, F. (2018c). Relationship between Foo Length Measurements and Body Height: A Prospective Regional Study among Adolescents in Central Region of Kosovo. Journal of Contemporary Medical Sciences, in press

Masanovic, B., Gardasevic, J., \& Arifi, F. (2018d). Standing Height and its Estimation Utilizing Foot Length Measurements in Adolescents from Southern Region in Kosovo. Sport Mont, 16(2), 27-31.

Masanovic, B., Gardasevic, J., \& Arifi, F. (2018e). Sitting Height/Standing Height Relationship in Southern Region of Kosovo. Retrieved from SSRN's eLibrary: https://ssrn.com/abstract $=3138523$

Masanovic, B., Gardasevic, J., \& Arifi, F. (2018f). Sitting Height/Standing Height Relations in Central Region of Kosovo. Retrieved from SSRN's eLibrary: https://ssrn.com/abstract=3138525

Masanovic, B., Gardasevic, J., \& Arifi, F. (2018g). Sitting Height/Standing Height Relationship Measurements in Northern Region of Kosovo. Retrieved from SSRN's eLibrary: https://ssrn.com/abstract=3138526

Menezes, R. G., Kanchan, T., Kumar, G. P., Rao. P. P., Lobo, S. W., Uzsal, S.,Krishan, K., Kalthur, S. G., Nagesh, K. R., \& Shettigar, S. (2009). Stature estimation from the length of the sternum in South Indian males: a preliminary stady. J. Formensic Leg. Med. 16(8), 441-3.

Milašinović, R, Gardašević, J, \& Bjelica, D. (2017). Body height and its estimation utilizing arm span measurements in male adolescents from northern region in Montenegro. Arta Kinesiologica, 11(2), 75-80.

Milašinović, R., Popović, S., Bjelica, D., \& Vasiljević, I. (2016). Body height and its estimation utilizing arm span measurements in female adolescents from northern region in Montenegro. In Book of Abstracts of 4th International Scientific Conference "Exercise and Quality of Life" (39), Novi Sad: Faculty of Sport and Physical Education.

Nagesh, K. R., \& Pradeep Kumar, G.(2006). Estimation of stature from verte- bral column length in South Indians. Leg. Med. 8(5):269-72.

Pineau, J. C., Delamarche, P., \& Bozinovic, S. (2005). Average height of adolescents in the Dinaric Alps. C. R. Biol., 328(9), 841-6.

Popovic, S. \& Bjelica, D. (2016). Body Height and its Estimation Utilizing Arm Span Measurements in Kosovan Adolescence: National Survey. In Abstract Book of International Eurasian Conference on Sport, Education, and Society (9), Antalya: International Science Culture and Sport Association.

Popovic, S. (2016). Body Height and its Estimation Utilizing Arm Span Measurements in Montenegrin Adults: National Survey. In Book of Summaries of 11th FIEP European Congress "Anthropological Aspects of Sport, Physical Education and Recreation" (5-6), Banjaluka: University of Banjaluka, Faculty of Physical Education and Sport.

Popovic, S. (2017). Local Geographical Differences in Adult Body Height in Montenegro. Montenegrin Journal of Sports Science and Medicine, 6(1), 81-7.

Popovic, S. Gardasevic, J., Masanovic, B., Arifi, F., \& Bjelica, D. (2017a). Standing Height and its Estimation Utilizing Foot Length Measurements in Adolescents from Western Region in Kosovo. Sport Mont, 15(3), 3-7.

Popovic, S., \& Bjelica, D. (2017). Body Height and its Estimation Utilizing Foot Length Measurementsin Kosovan Adults: National Survey. In Abstract Book of the Sport Science Conference AESA 2017(2), Amol: Faculty of Sport Sciences, Shomal University; Asian Exerciseand Sp Science Association.

Popovic, S., Arifi, F., \& Bjelica, D. (2017). Standing Height and its Estimation Utilizing Foot Length Measurements in Kosovan Adults: National Survey. International Journal of Applied Exercise Physiology, 6(2), 1-7.

Popović, S., Bjelica, D., Milašinović, R., \& Gardašević, J. (2016). Body height and its estimation utilizing arm span measurements in male adolescents from northern region in Montenegro. In Book of Abstracts of 4th International Scientific Conference "Exercise and Quality of Life" (38), Novi Sad: Faculty of Sport and Physical Education.

Popović, S., Bjelica, D., Milašinović, R., Gardašević, J., \& Rašidagić, F. (2016). Body height and its estimation utilizing arm span measurements in male adolescents from Herzeg-Bosnia entity in Bosnia and Herzegovina. In Book of Abstracts of IUAES Inter Congress "World anthropologies and privatization of knowledge: engaging anthropology in public" (148), Dubrovnik: International Union of Anthropological and Ethnological Sciences.

Quanjer, P.H., Capderou, A., Mazocioglu, M.M., Aggarwal, A., Popovic, S., Datta Banik, S., Tayie, F.A.K., Golshan, M., Ip, M.S.M., \& Zelter, M. (2014). All-age relationship between arm span and height in different ethnic groups. European Respiratory Journal, 44(4), 905-912.

Vukotic, M. (2018). Body height and its estimation utilizing arm span measurements in male and female adolescents from Danilovgrad and Cetinje. Journal of Anthropology of Sport and Physical Education, 2(3), 117121. doi: $10.26773 /$ jaspe. 180720 